\title{
An interesting case report of a giant bladder calculi in China
}

\author{
Chen Hui ${ }^{12^{2 *}}$, Huang Maping ${ }^{1}$, Huang Tianhai ${ }^{1}$, Yang Xiaoyi ${ }^{1}$, Ni $\mathrm{JJ}^{1}$, Li Qingqing ${ }^{1}$, Liu Qiuling ${ }^{1}$, Jiang Chonghe ${ }^{2}$ and Xie Keji ${ }^{3}$ \\ ${ }^{1}$ Department of Urology, Guangdong Provincial Work Injury Rehabilitation Hospital and Jinan University, Guangzhou, 510440, China \\ ${ }^{2}$ Department of Urology, Qingyan City People's Hospital, Guangdong, 511500, China \\ ${ }^{3}$ Department of Urology, Guangzhou First Municipal People’s Hospital, Guangzhou, 510180, China
}

\begin{abstract}
Objective: To report a giant bladder calculi in a 52 year old man.

\section{Introduction}

Bladder calculi is an old and rare disease which accounted for $5 \%$ of all urinary calculi $[1,2]$. Nowadays ,based on technical improvements and a growing clinical expertise on minimally invasive treatment, a giant stone in bladder is a very rare condition in china during the past twenty years. In this report, we reported a uncommon case of large bladder calculi.
\end{abstract}

Methods: The patient was diagnosed as bladder calculi since 1998. Due to the lack of sufficient medical and health conditions in the countryside, he only received the Chinese traditional treatment and did not seek any urologic treatments for more than 20 years. In April 2018, he was referred to our hospital with dysuria.

Results: A big bladder calculi $(8.0 \times 10.0 \times 6.0 \mathrm{~cm}$ in size $)$ was revealed by ultrasound and intravenous pyelography and removed by open cystolithotomy.

Conclusions: Our case demonstrates the necessity of early treatment for patients with bladder calculi.

\section{Case report}

In February 2018, a 52-year-old man was admitted to hospital due to a complaint of intermittent, paiful voiding, terminal hematuria and interruption of the urinary stream. The patient's medical history revealed that some of these typical worsening discomfort had been observed twenty years prior to the present study. Due to the lack of sufficient medical and health conditions in the countryside, he only received the Chinese traditional treatment and did not seek any urologic treatments for more than 20 years.

Systemic physical examination and rectal examination findings were normal. A complete urologic examination revealed a palpable solid oval-shaped mass, $8 \mathrm{~cm}$ in size, which originated from suprapubic region of the bladder. Both Color Doppler ultrasound of urinary system and intravenous pyelography(IVP) produced that a large, rounded calcified mass in the pelvis (Figure 1). Undoubtedly, it is difficult for endoscopic techniques to remove such a giant bladder calculi completely. Hence, open cystolithotomy was performed (Figure 2). More interestingly, although the bladder calculi size was relatively huge, it was removed easily and completely without adhesion to the bladder wall. The extirpated bladder calculi measured $8.0 \times 10.0 \times 6.0$ $\mathrm{cm}$, and weighed 293.1 gram (Figures 3 and 4). The postoperative 3 months follow-up was uneventful and without any urologic complaint. The calculus was analysed and found to be composed of cystine.

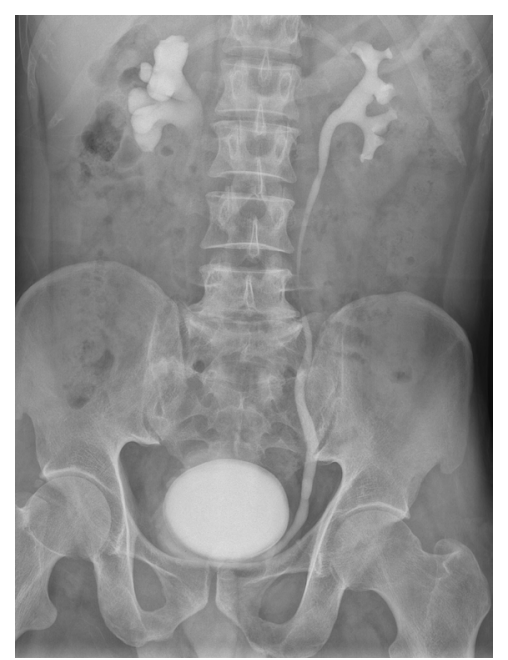

Figure 1. Showing a giant bladder calculus in the pelvic area

\section{Discussion}

It is well recognized that bladder stone usually result from bladder outlet obstruction, neurogenic voiding dysfunction, or recurrent urinary tract infection [3]. Bladder calculi are usually solitary but may

${ }^{*}$ Correspondence to: Chen Hui, Department of Urology, Guangdong Provincial Work Injury Rehabilitation Hospital and Jinan University; Department of Urology, Qingyan City People's Hospital, Guangzhou, 510440, China, E-mail: doc.chenhui@163.com

Key words: giant, bladder calculi

Received: October 08, 2018; Accepted: October 26, 2018; Published: October 29,2018 


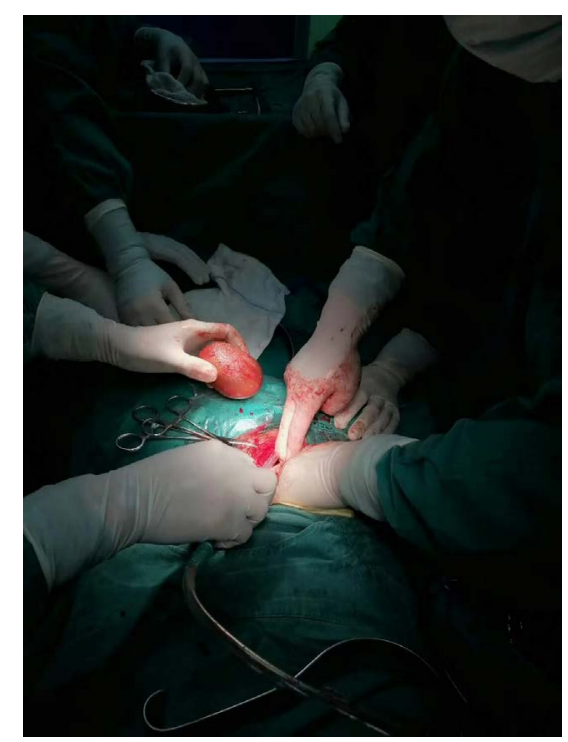

Figure 2. The giant bladder calculi was removed from bladder

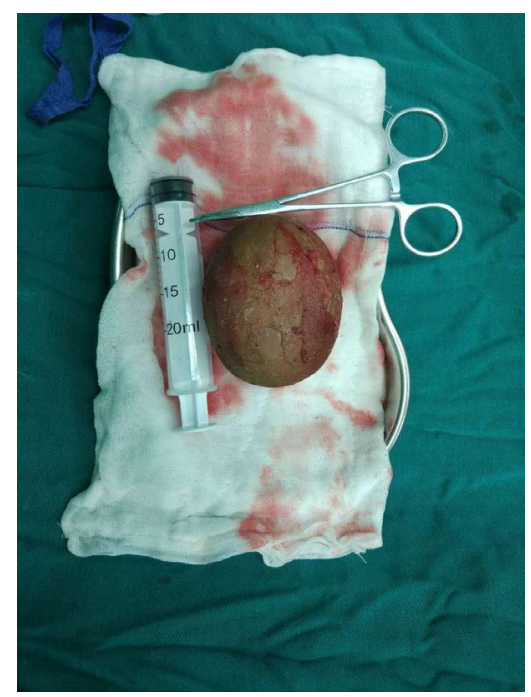

Figure 3. An intraoperative photograph showing the bladder calculi

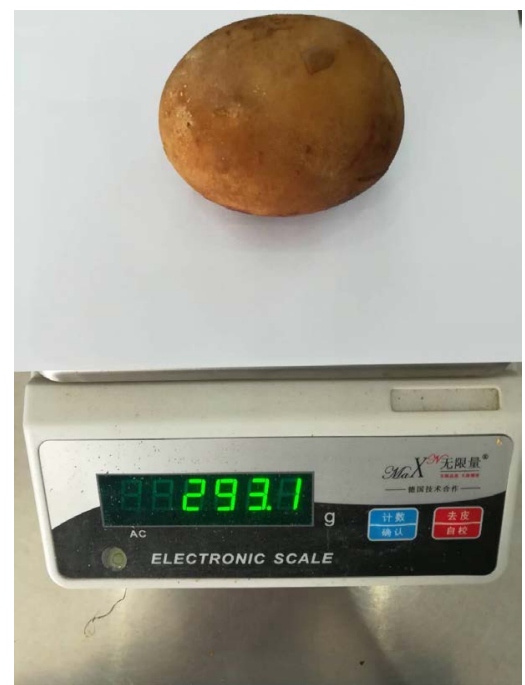

Figure 4. The extirpated calculi weighed 293.1gram develop in multiple stones in the presence of urinary stasis [4]. With the rapid development of China's economy and health care, bladder stones weighing more than $100 \mathrm{~g}$ are extremely rare. Undoubtedly, the present case report was more interesting. Firstly, a review of the literature shows that similar studies of giant bladder stone in china are scanty. Secondary, the extremely large bladder calculi occupied most of the bladder and pressing on the orifices of the ureters, leading to the presence of hydronephrosis. Thirdly, the most important point was that we found no obstructed bladder outlet during the operation. The reasons we analyzed were that the bladder stone formed after an upper urinary tract calculi descended into the bladder a long time ago.

Nowadays, it was wellknown that open approach has been progressively replaced by newer techniques aimed to be less invasive [5]. However, irrespective of a large number of alternative surgical procedures available today, we still choose open cystolithotomy as treatment choices for this case because it ensured wide vision of the surgical field to remove such a larger bladder calculi more rapidly and entirely. Fortunately, the bladder calculi was smooth and not adherent to the bladder wall which help avoid hematuria, bladder perforations and mucosal injuries risk.

Bladder calculi is associated with chronic irritation and inflammation which play a direct role in the development of bladder cancer [6,7]. In 2008, Farouk $\mathrm{K}$ and his colleague [8] reported a 65 -year-old man with bladder leiomyoma and bladder stone. In 2013, another study [9] even reported that bladder calculi was found up to 71 (3.4\%) cases in 2,086 patients with bladder cancer between 2001 and 2009. Fortunately, in this case, the interior of tha patient bladder was normal and no neoplasm was visible to the naked eyes after the stone was removed.

\section{Conclusion}

This case study reported satisfactory results for a giant stone in 2-year-old male patient. However, bladder stone should be taken into consideration when patients develop lower urinary tract symptoms or other signs occur, such as intermittent, painful voiding, terminal hematuria recurrent urinary tract infection and interruption of the urinary stream.

\section{Acknowledgments}

This study was supported by Medical Scientific Research Foundation of Guangdong Province, China (grant number A2013477, A2018124).

\section{Conflicts of interest}

The authors declare no conflict of interest.

\section{References}

1. Sorokin I, Mamoulakis C, Miyazawa K, Rodgers A, Talati J, et al. (2017) Epidemiology of stone disease across the world. World J Urol 35: 1301-1320. [Crossref]

2. Schwartz BF, Stoller ML (2000) The vesical calculus. Urol Clin North Am 27: 333-346 [Crossref]

3. Türk C, Petřík A, Sarica K, Seitz C, Skolarikos A, et al. (2016) EAU guidelines on interventional treatment for urolithiasis. Eur Urol 69: 475-482. [Crossref]

4. Torricelli FC, Chueh SJ, Shen S, Monga M (2017) Multiple uric acid bladder stones: Clinical presentation and endoscopic management. J Endourol Case Rep 3: 21-23. [Crossref]

5. Cicione A, DE Nunzio C, Manno S, Damiano R, Posti A, et al. (2018) Bladder stone management: an update. Minerva Urol Nefrol 70: 53-65. [Crossref] 
6. Chamie K, Litwin MS, Bassett JC, Daskivich TJ, Lai J, et al. (2013) Recurrence of highrisk bladder cancer: a population-based analysis. Cancer 119: 3219-3227. [Crossref]

7. Nesi G, Nobili S, Cai T, Caini S, Santi R (2015) Chronic inflammation in urothelial bladder cancer. Virchows Arch 467: 623-633. [Crossref]
8. Farouk K, Gondal M, Ahmad A, Bano U, Khan A (2008) Leiomyoma of the urinary bladder with bladder stone. J Coll Physicians Surg Pak 18: 592-594. [Crossref]

9. Chung SD, Tsai MC, Lin CC, Lin HC (2013) A case-control study on the association between bladder cancer and prior bladder calculus. BMC Cancer 13: 117. [Crossref]

Copyright: (C2018 Hui C. This is an open-access article distributed under the terms of the Creative Commons Attribution License, which permits unrestricted use, distribution, and reproduction in any medium, provided the original author and source are credited. 